Research Article

\title{
Comparison of Open Gastrostomy and Percutaneous Endoscopic Gastrostomy in Head and Neck Cancer Patients, Tertiary Care Hospital Experience
}

\author{
Talha Ahmed Qureshi ${ }^{*}, 1$, Shabir Akhtar², Sohail Awan², Amir Shariff ${ }^{3}$, Mohammad Adeel $^{4}$ \\ ${ }^{1}$ Section of Otolaryngology, Department of Surgery, Patel Hospital, Karachi, Pakistan. \\ ${ }^{2}$ Section of Otolaryngology, Department of Surgery, Aga Khan Hospital, Karachi, Pakistan. \\ ${ }^{3}$ Department of Surgery, Aga Khan Hospital, Karachi, Pakistan. \\ ${ }^{4}$ Clinical Fellow, Bradford Royal Infirmary, Bradford, United Kingdom.
}

\begin{abstract}
Objective: The current study was conducted to review and compare PEG and Gastrostomy in terms of early complications in head and neck cancer patients.

Materials and Methods: This was prospective comparative study recruited total 60 patients as per the inclusion criteria, 30 in each group. Patients were assigned to the groups based on the surgeon's choice. Ethical clearance was taken from ethical review board. Informed consent was taken from patients before enrolling them into the study. Non-probability consecutive sampling technique was used to enroll study participants. Data was collected on pre-designed performa and analyzed using SPSS version 16.
\end{abstract}

Setting: Section of Otolaryngology, Department of Surgery, Aga Khan University Hospital Karachi.

Results: The two groups did not differ on basis of age, gender and tumor site. The outcomes variables including pain and bleeding from post-operative day 1 to day 5 were significantly lower in patients who underwent PEG placement as compared to patients whom we performed OPEN gastrostomy. None the patient had peristomal infection in PEG group throughout the study. However, difference between the groups was statistically significant from post-operative day 3 to day 5 .

Conclusion: In our study, PEG was associated with lesser complications than open gastrostomy. Recently clinicians are using newer modalities like radiological and laparoscopic gastrostomy at various centres in Pakistan as the first option for placing feeding gastrostomy. Therefore, future studies are warranted to compare their efficacy and post-operative complications PEG.

Keywords: Head and neck cancer, Open gastrostomy, Percutaneous endoscopic gastrostomy, Complications, Pain, Peristomal infection, Bleeding.

\section{INTRODUCTION}

Among all the tumor related cases, Head and Neck Squamous cell carcinoma (HNSCC) makes 600,000 cases in universe accounting or $40-50 \%$ yearly deaths. Moreover, the burden of two-folds is expected in low and middle income countries by the end of 2030 [1]. Globally, head and neck tumors stands as sixth most occurring cancer cases and $25 \%$ of these cases are reported from South Asian countries [2]. In Pakistan, it is observed as second most frequent malignancy among adult males [3].

There are also various modalities for the treatment of head and neck tumors. Patients with malignancies of HNC including oral cavity, larynx or pharynx usually treated with or without combination of surgical resection of tumor with radiotherapy and/or chemotherapy [4]. About two-third of HNC cases present with dysphagia and three-fourth treated patients develop it [5]. Trismus, the condition of restricted mouth opening is also frequent in HNC patients which limits speaking, swallowing and eating [6]. Owing to these factors,

*Address correspondence to this author at the Section of Otolaryngology, Department of Surgery, Patel Hospital, Karachi, Pakistan.

Email: tal_qureshi@yahoo.com patients with head and neck cancer (HNC) are at high risk of malnutrition and estimated malnutrition prevalence of $35-60 \%$ is reported in $\mathrm{HNC}$ patients receiving radiotherapy [7].

Schadev et al reported in his study that $33 \%$ of HNC cancer required enteral feeding tube insertion following the radiation therapy [8]. Multiple interventions have been designed for improving the nutritional status for the patients and control the problem of weight loss. These interventions are nutritional supplements, counseling sessions for improved diet and placement of percutaneous endoscopic gastrostomy (PEG) tube [7].

Gastrostomy is a conservative modality for feeding through tube for purpose of medication, hydration and nutritional enhancement. Stamm firstly introduced open surgical gastrostomy (OSG) in 1894. OSG was considered as the standard procedure for gastric decompression and enteral access prior to early 1980 's, when PEG was launched and since then PEG has been considered as the preferred procedure over OSG owing to its affordability and lesser postoperative complications rate $[9,10]$.

www.njhsciences.com 
Endoscopic gastrostomy is beneficial in numerous ways. In contrast to the OSG, it not merely eradicate the requirement of operating room, but also associated with reduced complication rate, procedure time and expense $[5,6]$. Conversely, endoscopic gastrostomy is not viable all the time, usually when it can't be performed in HNC patients causing obstruction, or if diaphanoscopy of the stomach is not achieved. In such cases, some other technique is required for gastrostomy. Hence, feeding through PEG or OSG is a debatable topic. However, to the best of our knowledge, there are no local studies available that studied efficacy of these two techniques. Therefore, we planned a present study to compare complications PEG versus OSG in our local settings.

\section{MATERIALS AND METHODS}

The current prospective comparative study was conducted at Section of Otolaryngology, Department of Surgery, Aga Khan University Hospital Karachi.

Patients fulfilling inclusion /exclusion criteria and agreeing to sign informed consent were enrolled into study. Ethical clearance was taken from Ethical Review Board. Patients were enrolled through non-probability consecutive sampling. According to the primary surgeon's preference, patients were assigned to either OSG or PEG group.

\section{SAMPLE SIZE}

Complication rate after open gastrostomy and percutaneous endoscopic gastrostomy has been reported up to $17.1 \%$ and $5 \%$ respectively (in terms of tube dislodgement which has the minimum reported frequency). With $90 \%$ confidence level and bound error of $9 \%$ sample size calculated is 32 for each group [11].

\section{INCLUSION CRITERIA}

All patients in both genders with age range 16-60 years having gastrostomy tube placed at the time of primary head and neck surgery in our institute.

\section{EXCLUSION CRITERIA}

- All head and neck cancer patients who did not have gastrostomy.

- All head and neck cancer patients who had gastrostomy done in a second setting.

- History of abdominal trauma.

- History of abdominal surgery.

- History of narcotic addiction.

Age, gender, primary lesion, and days of hospitalization were recorded. Primary endpoint was pain at stomal site on visual analogue score (VAS), bleeding and leakage in terms of number of gauzes soaked, presence or absence of tube dislodgement and peristomal erythema was considered as an indicator of infection (rated on a scale of 1-5 for every $5 \mathrm{~mm}$ of erythema).

\section{DATA ANALYSIS}

Data were stored and analyzed using SPSS 16.0, Mean and standard deviation were calculated for age, pain scores, and bleeding and leakage. Independent sample t-test was used to compare the mean differences between two study groups, while Pearson chi square test was used to see the association of type with gender, lesions and other qualitative variables. P-values less than 0.05 were considered as statistically significant.

\section{RESULTS}

Patients' demographic variables are depicted in Table $\mathbf{1}$. Mean age of patients who had open gastrostomy and PEG was $45.86 \pm 11.36$ years and $50.1 \pm 12.67$ respectively. Male proportion was $26(86.7 \%)$ and $25(83.3 \%)$ for open and PEG groups respectively. The two groups didn't differ on the basis of age, gender and lesions.

Table 1. Comparison of Patients' Characteristics between PEG and OPEN.

\begin{tabular}{|c|c|c|c|}
\hline Variables & $\begin{array}{l}\text { PEG } \\
\text { n(\%) }\end{array}$ & $\begin{array}{c}\text { OPEN } \\
\mathbf{n}(\%) \\
\end{array}$ & p-value \\
\hline Age $^{\#}$ (in years) & $50.1 \pm 12.67$ & $45.86 \pm 11.36$ & 0.17 \\
\hline \multicolumn{4}{|l|}{ Gender } \\
\hline Male & $25(83.3)$ & $26(86.7)$ & \multirow{2}{*}{1.00} \\
\hline Female & $5(16.7)$ & $4(13.3)$ & \\
\hline \multicolumn{4}{|l|}{ Lesion } \\
\hline Tongue & $2(6.7)$ & $6(20)$ & \multirow{11}{*}{0.227} \\
\hline Buccal mucoa & $17(56.7)$ & $16(53.3)$ & \\
\hline Retromolar triagone & $1(3.3)$ & $0(0)$ & \\
\hline Floor of mouth & $1(3.3)$ & $1(3.3)$ & \\
\hline Upper alveolus & $0(0)$ & $1(3.3)$ & \\
\hline Lower alveolus & $3(10)$ & $0(0)$ & \\
\hline Hard palate/maxilla & $1(3.3)$ & $2(6.7)$ & \\
\hline Oropharynx & $1(3.3)$ & $0(0)$ & \\
\hline Hypopharynx & $0(0)$ & $2(6.7)$ & \\
\hline Larynx & $1(3.3)$ & $0(0)$ & \\
\hline Multiple/complex & $3(10)$ & $2(6.7)$ & \\
\hline
\end{tabular}

\#: Indicating variable is expressed as mean \pm standard deviation, PEG: Percutaneous endoscopic gastrostomy; OPEN: Open gastrostomy.

Study outcomes are compared between PEG and open gastrostomy groups in Table $\mathbf{2}$. Mean pain score at post-opera- 
tive from day 1 to day 5 was significantly higher in open open gastrostomy group as compared to PEG. Significant difference was seen between the study groups in terms of peristomal bleeding or leakage during post-operative day 1 till day 5 . Bleeding or leakage was significant in the open gastrostomy group than in the PEG group but not statistically significant in terms of difference in number of gauzes soaked. Anatomical sub-sites were observed and $56.7 \%$ of patients in the PEG and $53.3 \%$ patients in the open gastrostomy group had buccalmucosal lesions. Peristomal infection was not observed in PEG group. It was only observed in open gastrostomy patients from day 1 to day 5 post-operative. However, difference between the groups was statistically significant from post-operative day 3 to day 5 .

Table 2. Comparison of Study Outcomes between Two Groups.

\begin{tabular}{|c|c|c|c|}
\hline Variables & $\begin{array}{l}\text { PEG } \\
\text { n(\%) }\end{array}$ & $\begin{array}{c}\text { OPEN } \\
\text { n(\%) }\end{array}$ & p-value \\
\hline TAD & $6.43 \pm 1.86$ & $6.33 \pm 2.15$ & 0.84 \\
\hline Pain on post-operative day $1^{\#}$ & $1.96 \pm 0.92$ & $6.1 \pm 0.95$ & $* *<0.01$ \\
\hline Pain on post-operative day $2^{\#}$ & $1.53 \pm 0.81$ & $5.33 \pm 1.15$ & $* *<0.01$ \\
\hline Pain on post-operative day $3^{\#}$ & $1 \pm 0.72$ & $4.53 \pm 1.3$ & $* *<0.01$ \\
\hline Pain on post-operative day $4^{\#}$ & $0.71 \pm 0.64$ & $3.22 \pm 1.01$ & $* *<0.01$ \\
\hline Pain on post-operative day $5^{\#}$ & $0.3 \pm 0.48$ & $2.66 \pm 1.11$ & $* *<0.01$ \\
\hline BL on post-operative day $1^{\#}$ & $1.2 \pm 0.4$ & $1.56 \pm 0.62$ & $* *<0.01$ \\
\hline BL on post-operative day $2^{\#}$ & $1.03 \pm 0.18$ & $1.46 \pm 0.57$ & $* *<0.01$ \\
\hline BL on post-operative day $3^{\#}$ & $1 \pm 0.01$ & $1.4 \pm 0.56$ & $* *<0.01$ \\
\hline BL on post-operative day $4^{\#}$ & $1 \pm 0.01$ & $1.33 \pm 0.48$ & $* *<0.01$ \\
\hline BL on post-operative day $5^{\#}$ & $1 \pm 0.01$ & $1.55 \pm 0.52$ & $* *<0.01$ \\
\hline \multicolumn{4}{|l|}{ TD } \\
\hline Yes & $0(0)$ & $1(100)$ & \multirow{2}{*}{1.00} \\
\hline No & $30(50.8)$ & $29(49.2)$ & \\
\hline \multicolumn{4}{|l|}{ PI on post-operative day 1} \\
\hline None & $30(50.8)$ & $29(49.2)$ & \multirow{2}{*}{0.31} \\
\hline $5 \mathrm{~mm}$ & $0(0)$ & $1(100)$ & \\
\hline \multicolumn{4}{|l|}{ PI on post-operative day 2} \\
\hline None & $30(50.8)$ & $29(49.2)$ & \multirow{2}{*}{0.31} \\
\hline $5 \mathrm{~mm}$ & $0(0)$ & $1(100)$ & \\
\hline \multicolumn{4}{|l|}{ PI on post-operative day 3} \\
\hline None & $28(53.8)$ & $24(46.2)$ & \multirow{2}{*}{$* 0.01$} \\
\hline $5 \mathrm{~mm}$ & $0(0)$ & $6(100)$ & \\
\hline \multicolumn{4}{|l|}{ PI on post-operative day 4} \\
\hline None & $21(55.3)$ & $17(44.7)$ & \multirow{2}{*}{$* 0.02$} \\
\hline $5 \mathrm{~mm}$ & $0(0)$ & $10(100)$ & \\
\hline \multicolumn{4}{|l|}{ PI on post-operative day 5} \\
\hline None & $13(76.5)$ & $4(23.5)$ & \multirow{2}{*}{$* *<0.01$} \\
\hline $5 \mathrm{~mm}$ & $0(0)$ & $4(100)$ & \\
\hline
\end{tabular}

BL: Bleeding or leakage, PEG: Percutaneous endoscopic gastrostomy, OPEN: Open gastrostomy, TAD: Total admission day, TD: Tube dislodgement, PI: Peristomal infection.

\#: Indicating variable is expressed as mean \pm standard deviation, *Significant at $\mathrm{P}<0.05$, ** Significant at $\mathrm{P}<0.01$.

\section{DISCUSSION}

Globally, some of the healthcare centers try to avoid PEG tube insertion unless the onset of weight loss and/or swallowing disorders whereas some facility providers prophylactically inert it prior to treatment. Internationally, there are such acceptable standardized methods to assess nutritional condition of cancer patients to determine the use of prophylactic use of PEG. The guidelines issued by European Society for Clinical Nutrition and Metabolism (ESPEN) emphasized that malnutrition in times of cancer management leads to poor quality of life, increases side-effects and lessen response to the treatment $[10,12]$. As per the general criteria, patients with insufficient nutritional intake for at least two weeks should be considered as candidates of gastrostomy placement [13]. A feeding gastrostomy tube is an effective method for obtaining enteral access among patients who are unable to swallow and hence not meeting the sufficient nutritional needs.

Worldwide, OSG has replaced PEG in almost all of the healthcare centers and PEG has become treatment of choice [14-16]. PEG is less-invasive procedure OSG and may be performed through endoscopy, sonography or fluoroscopy. Studies have been conducted around the world to compare outcomes for PEG and OSG. It has been reported in previously available literature that OSG accounts for higher complications and mortality as it is an invasive procedure which require longer recover time. Furthermore, OSG is more costly and requires operating room reservation and anesthesia team in all of the cases and some of the patients also require intensive care $[17,18]$.

In the present study, we identified the significant reduced post-operative pain excluding for day 1 only for patients receiving PEG. Moreover, there was also significant reduction in bleeding or leakage from day 1 to day 5. PEG was also observed to be superior to $\mathrm{OG}$ in terms of complications as peristomal infection was not observed among none of the patients belonging to PEG group from day 1 to day 5. However, significance was observed from day 3. According to Lai and co-authors, percutaneous endoscopic gastrostomy (PEG) is a harmless and minimally invasive technique that can be conducted in most patients to achieve feeding access. There is no necessary requirement of operating room in most of PEG placements, and the procedure may be conducted either in the endoscopy suite (wherever moderate sedation is permitted) or intensive care unit. There is only $10 \%$ chance of associated complications which are frequently minor in nature. They also emphasized that open gastrostomy is a second option in cases 
where placement of PEG is not possible [19]. Arising literature is in support of endoscopic gastrostomy tube placement over surgical gastrostomy because of its less invasive nature and faster time to start feeding [20].

\section{LIMITATIONS}

The limitations of the present study is the small sample size i.e. 30 patients in each group. Secondly, the follow-up time was shorter so we could not observe the long-term complications or mortality rate between the two groups. A prospective future study is suggested with larger sample size to confirm the superiority of PEG to OG with larger follow-up duration to address the long-term outcomes

\section{CONCLUSION}

In our study, PEG was associated with lesser complications than open gastrostomy. Clinicians are using different modalities in Pakistan for placing feeding gastrostomy. Therefore, future studies are warranted to compare their efficacy and post-operative complications PEG.

\section{AUTHORS' CONTRIBUTION}

Talha Quershi: Had a main idea of the study, searched literature and drafted the article.

Shabbir Akhtar: Contributed in data analysis and manuscript writing.

Sohail Awan: Helped in editing manuscript.

Amir Sharif: Helped in searching literature and manuscript writing.

Mohammad Adeel: Helped with analysis.

\section{CONFLICT OF INTEREST}

Declared none.

\section{ACKNOWLEDGEMENTS}

Authors are thankful to Ms. Maria Baig, Editorial Manager of National Journal of Health Sciences for her guidance and support in providing us the guidelines for preparing and formatting of the manuscript.

\section{REFERENCES}

[1] Gupta B, Johnson NW, Kumar N. Global epidemiology of head and neck cancers: A continuing challenge. Oncology 2016; 91(1): 13-23. DOI: 10.1159/000446117

[2] Khan NF, Saeed M, Butt AK, Khan AA. Epidemiology of head and neck Neoplasm's in Balochistan. J Pak Dent Assoc 2017; 26(03): 119. DOI: 10.25301/JPDA.263.118

[3] Akhtar A, Hussain I, Talha M, et al. Prevalence and diagnostic of head and neck cancer in Pakistan. Pak J Pharm Sci 2016; 29(5 Suppl): 1839-46.

[4] Alsahafi E, Begg K, Amelio I, et al. Clinical update on head and neck cancer: Molecular biology and ongoing challenges. Cell Death Dis 2019; 10(8): 1-17. DOI: 10.1038/s41419-019-1769-9

[5] Retes FA, Kawaguti FS, de Lima MS, et al. Comparison of the pull and introducer percutaneous endoscopic gastrostomy techniques in patients with head and neck cancer. United Eur Gastroenterol J 2017; 5(3): 365-73. DOI: $10.1177 / 2050640616662160$

[6] Loh SY, Mcleod RW, Elhassan HA. Trismus following different treatment modalities for head and neck cancer: A systematic review of subjective measures. Eur Arch Otorhinolaryngol 2017; 274(7): 2695-707. DOI: 10.1007/s00405-017-4519-6

[7] Lang K, ElShafie RA, Akbaba S, et al. Percutaneous endoscopic gastrostomy tube placement in patients with head and neck cancer treated with radiotherapy. Cancer Manag Res 2020; 12: 127. DOI: 10.2147/CMAR.S218432

[8] Sachdev S, Refaat T, Bacchus ID, Sathiaseelan V, Mittal BB. Age most significant predictor of requiring enteral feeding in head-and-neck cancer patients. Radiat Oncol 2015; 10(1): 93. DOI: 10.1186/s13014-015-0408-6

[9] Mizrahi I, Garg M, Divino CM, Nguyen S. Comparison of laparoscopic versus open approach to gastrostomy tubes. JSLS 2014; 18(1): 28. DOI: 10.4293/108680813X13693422520927

[10] Langmore S, Krisciunas GP, Miloro KV, Evans SR, Cheng DM. Does PEG use cause dysphagia in head and neck cancer patients? Dysphagia 2012; 27(2): 251-9. DOI: $10.1007 / \mathrm{s} 00455-011-9360-2$

[11] Ljungdahl M, Sundbom M. Complication rate lower after percutaneous endoscopic gastrostomy than after surgical gastrostomy: A prospective, randomized trial. Surg Endoscopy 2006 ; 20(8): 1248-51. DOI: $10.1007 / \mathrm{s} 00464-005-0757-6$

[12] Arends J, Bachmann P, Baracos V, et al. ESPEN guidelines on nutrition in cancer patients. Clin Nutr 2017; 36(1): 11-48. DOI: 10.1016/j.clnu.2016.07.015

[13] Löser C, Aschl G, Hebuterne X, et al. ESPEN guidelines on artificial enteral nutrition--percutaneous endoscopic gastrostomy (PEG). Clin Nutr 2005; 24(5): 848-61. DOI: 10.1016/j.clnu.2005.06.013

[14] Gauderer MW, Ponsky JL, Izant RJ. Gastrostomy without laparotomy: A percutaneous endoscopic technique. J Pediatr Surg 1980; $15(6)$ : $\quad 872-5$. 10.1016/S0022-3468(80)80296-X

[15] Sacks D, McClenny TE, Cardella JF, Lewis CA. Society of interventional radiology clinical practice guidelines. J Vasc Interv Radiol 2003; 14(9 Pt 2): S199-202. DOI: 10.1097/01.RVI.0000094584.83406.3e

[16] Zopf Y, Maiss J, Konturek P, Rabe C, Hahn EG, Schwab D. Predictive factors of mortality after PEG insertion: guidance for clinical practice. J Parenter Enteral Nutr 2011; 35(1): 50-5. 
DOI: $10.1177 / 0148607110376197$

[17] Jones M, Santanello SA, Falcone RE. Percutaneous endoscopic vs. surgical gastrostomy. J Parenter Enteral Nutr 1990; 14(5): 533-4. DOI: 10.1177/0148607190014005533

[18] Leeds JS, McAlindon ME, Grant J, Robson HE, Lee FK, Sanders DS. Survival analysis after gastrostomy: a single-centre, observational study comparing radiological and endoscop- ic insertion. Eur J Gastroenterol Hepatol 2010; 22(5): 591-6. DOI: 10.1097/MEG.0b013e328332d2dd

[19] Lai L, Ali SF. Percutaneous endoscopic gastrostomy and open gastrostomy. Atlas Oral Maxillofac Surg Clin North Am 2015; 23(2): 165-8. DOI: 10.1016/j.cxom.2015.05.001

[20] Shah R, Shah M, Aleem A. Gastrostomy Tube Replacement. Treasure Island (FL): StatPearls Publishing 2021. 